\title{
Использование MS Excel при обработке пиков триацилглицеринов на хроматограммах растительных масел
}

\author{
(C) 2020 Дейнека В.И. ${ }^{1}$, Туртыгин А.В. ${ }^{2}$, Нгуен Ван Ань ${ }^{3}$, Дейнека Л.А. ${ }^{1}$ \\ ${ }^{I}$ ФГАОУ ВО «Белгородский государственный наииональный исследовательский университет», \\ Белгород \\ ${ }^{2}$ Федеральное автономное учреждение «Национальный Институт Аккредитации», Москва \\ ${ }^{3}$ Ханойский педагогический университет 2, Ханой, Вьетнам
}

Поступила в редакцию 27.02.2020 г.

DOI: $10.17308 /$ sorpchrom.2020.20/2780

Предложен способ определения видового состава триацилглицеринов (ТАГ) и жирнокислотного состава растительных масел с использованием инкрементного подхода с использованием простых и доступных компьютерных технологий - рабочего листа в стандартной программе MS Excel. Качественное определение основано на аддитивности вкладов фукнциональных групп (радикалов жирных кислот) в суммарное удерживание ТАГ и их независимости от строения двух остальных радикалов в рассматриваемой паре ТАГ. Для реализации инкрементного подхода предложен метод уточнения времени удерживания ТАГ при выходе за пределы линейности изотермы сорбции: в этом случае лучший вариант определения времени удерживания - положение вертикальной черты, делящей ширину пика на две равные части. В работе был использован способ определения «мертвого» времени по удерживанию серий псевдогомологов, - групп ТАГ с последовательной заменой радикалов одной кислоты на радикалы другой кислоты. Это было реализовано на примере персикового масла по временам удерживания ТАГ: $Л_{3}-Л_{2} \mathrm{O}-Л_{2}-\mathrm{O}_{3}$ по ранее предложенной схеме, основанной на рекуррентных соотношениях И.Г. Зенкевича. На основе анализа хроматограмм персикового масла были рассчитаны ключевые инкременты - для последовательно замены линолевой - олеиновой - пальмитиновой - стеариновой кислотами, использованные для расчета состава ТАГ масла семян нигеллы дамасской. Для количественного определения долей видов ТАГ использовали поправочные коэффициенты для экспериментальных значений площадей пиков вследствие зависимости чувствительности рефрактометрического детектора от разности коэффициентов преломления между подвижной фазой (экспериментальные данные) и коэффициентами преломления индивидуальных ТАГ, рассчитанными в ПО ChemSketch 12.0. Изложена процедура организации рабочего листа с указанием применяемых при расчетах формул. Метод использован для определения ТАГ и жирнокислотного состава масла семян Nigella damascene L., в результате чего было установлено, что масло содержит уникальную дигомолинолевую (C22:211Z14Z)

Ключевые слова: обращенно-фазовая ВЭЖХ, разделение триацилглицеринов, качественный и количественный анализ, рабой лист MS Excel.

\section{Введение}

Стандартные компьютерные программы (КП) для записи, хранения и обработки хроматограмм удобны, и они к настоящему времени практически полностью вытеснили специализированные самописцы. Эти программы содержат ряд важных в практике обработки хроматограмм функций. Но часто требуются дополнительные функции, специфичные для каждой конкретной задачи, решаемой оператором хроматографа. Так, например, время удерживания, начала и окончания пика определяются 
по производной сигнала детектора по времени, что приводит к ошибочным результатам при автоматической обработке хроматограммы в случае не полностью разделенных пиков. Разумеется, авторы КП это предвидели, и оператор имеет возможность провести корректировку обработки хроматограмм в таких случаях вручную. Но в реальной практике хроматографиста часто возникают проблемы, требующие решения, не предусмотренные программным обеспечением. Так при определении времени удерживания проблемы возникают при выходе за пределы линейности изотермы адсорбции на начальном участке разделения. Это явление характерно, например, для хроматографирования триацилглицеринов (ТАГ) растительных масел с рефрактометрическим детектором, чувствительность которого примерно на порядок меньше чувствительности спектрофотометрического детектора. Перегрузка колонки приводит к существенному искажению формы пика [1], и производная сигнала по времени обращается в ноль при временах, заметно отличающихся от времени удерживания тех же веществ при условиях линейности изотермы адсорбции. Вторая проблема относится к качественному и количественному определению веществ. При доступности стандартных образцов (CO) всех определяемых компонентов проблема решается градуировкой по этим $\mathrm{CO}$, но во многих случаях на стандартные образцы рассчитывать не приходится. К числу задач подобного типа относится обработка хроматограмм растительных масел, содержащих большое число индивидуальных ТАГ, СО которых недоступны.

Цель настоящей - разработка листа в программе MS Excel для необходимой и доступной дополнительной компьютерной обработки пиков ТАГ на хроматограммах растительных масел, включающих расчет времен удерживания для идентификации пиков, корректировку площадей для количественных расчетов долей ТАГ в масле и долей образовавших их жирных кислот.

\section{Эксперимент}

При разделении ТАГ растительных масел использовали хроматограф Shimadzu L20 с рефрактометрическим детектором; колонка 250×4.6 мм Kromasil 100-5C18; подвижная фаза $20 \%$ ацетонитрила в ацетоне, $0.8 \mathrm{~cm}^{3} /$ мин. Температура термостата колонок $30^{\circ} \mathrm{C}$.

Масла из семян экстрагировали $н$-гексаном в фарфоровой ступке при растирании. Порции $t$-гексана собирали в одну емкость, отфильтровывая от твердого остатка через бумажный фильтр. Полученный экстракт сорбировали на концентрирующем патроне Диапак С (БиоХимМак СТ). С патрона триацилглицерины десорбировали ацетоном и растворитель удаляли на вакуумном ротационном испарителе. Очищенные масла растворяли в растворителе, идентичном используемой подвижной фазы.

Триацилглицерины обозначали по общепринятой схеме - буквами указывали тип кислот с указанием их числа в ТАГ нижним индексом без дифференциации положения радикалов молекуле ТАГ. Буквенные обозначения четырех кислот, входящих в состав большинства растительных масел: Л - линолевая, О - олеиновая, П - пальмитиновая, С - стеариновая; буквами X, Y и др. обозначали специфические для масла кислоты. $Л_{2} \mathrm{O}$ обозначает ТАГ, образованный двумя радикалами линолевой и одним радикалом олеиновой кислоты.

Масло выделяли из семян: нигелла дамасская микс (ООО «АГРОНИ») и использовали без дополнительно очистки масло персиковое (ООО «Ботаника»).

Коэффициент преломления подвижной фазы измеряли на приборе ИРФ454Б2М. Коэффициенты преломления ТАГ рассчитывали, используя ChemSketch 12.0 (ACD). Неразделенные пики обрабатывали в программе MagicPlot Student. При обработке пиков использовали ПО: LCSolution, Shimadzu и MS Excel 2010. 


\section{Обсуждение результатов}

Первая проблема, которая должна быть решена для достижения поставленной цели - корректное определение времен удерживания. Дело в том, что при хроматографировании большой группы ТАГ при контроле элюата не отличающимся высокой чувствительностью рефрактометрическим детектором приходится использовать от-


к перегрузке колонки и сильному отклонению формы пика от гауссиана, рис. 1.

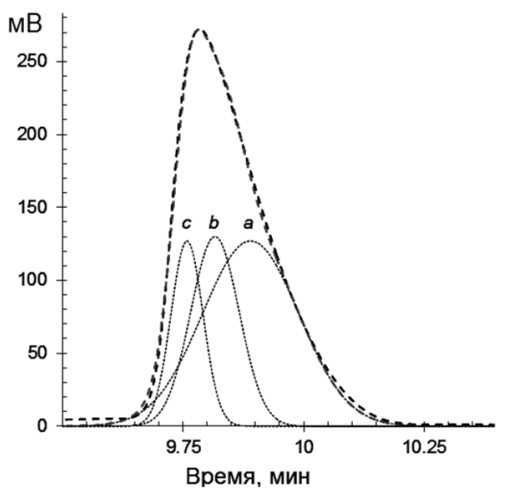

Рис. 1. Компьютерное моделирование искаженного из-за перегрузки пика тремя гауссианами

Пик три- $\alpha$-линоленоата при высокой стартовой концентрации льняного масла в образе $\left(10 \mathrm{мг} / \mathrm{cm}^{3}\right)$ весьма асимметричен и может быть представлен суммой не менее трех гауссианов. Но очевидно следующее: при выпуклой форме изотермы сорбции правая затянутая часть пика должна быть аппроксимирована гауссианом ( $a$, рис.1) с близкой крутизной спада сигнала детектора к сигналу, записанному при линейности изотермы адсорбции. А в данном случае этот компонент (a) имеет ширину, сопоставимую с шириной всего пика. Следовательно, лучшей оценкой времени удерживания будет время, соответствующее положению вертикали, делящей всю ширину искаженного пика на две равные части. Это подтверждается сопоставлением хроматограмм одного и того же масла (в данном случае - масла семян нигеллы дамасской), записанных при различных исходных концентрациях масла в пробе, рис. 2. Рис. 2 подтверждает то, что предложенный вариант определения времени удерживания согласуется с удерживанием ТАГ масел на хроматограммах, полученных при небольших концентрациях этих масел в пробе.

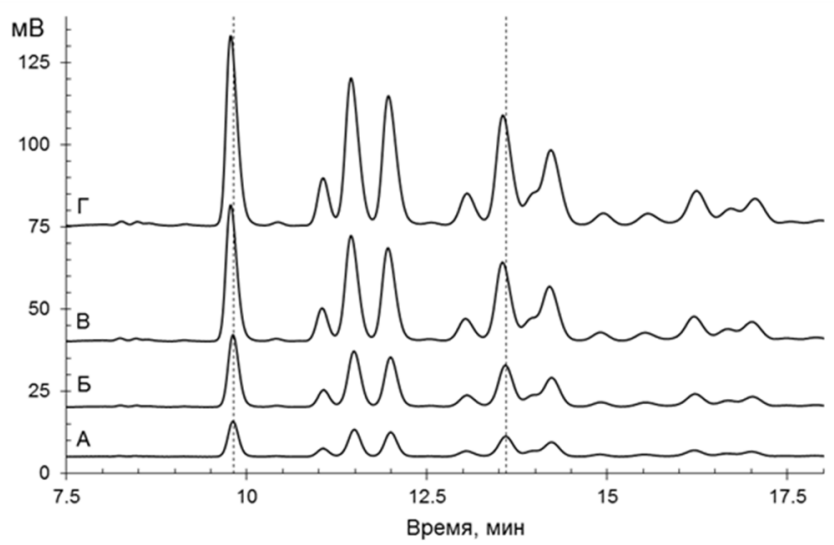

Рис. 2. Хроматограммы масел семян Nigella damascena

с различной концентрацией масла в пробе

Условия: колонка 250×4.6 мм, Kromasil 100-5C18, подвижная фаза, 20 об.\% ацетонитрила в ацетоне, $0.8 \mathrm{~cm}^{3} /$ мин. Концентрация: А - $5 \mathrm{мг} / \mathrm{cm}^{3} ;$ Б $-10 \mathrm{Mг} / \mathrm{cm}^{3}, \mathrm{~B}-20 \mathrm{Mг} / \mathrm{cm}^{3} ; \Gamma-30 \mathrm{Mг} / \mathrm{cm}^{3}$. 
Вторая проблема относительно качественного анализа состава ТАГ масел - отнесение пиков на хроматограмме. Для ее решения в нашей лаборатории был разработан инкрементный подход [2]. Инкрементный подход основан на идее аддитивности вкладов (инкрементов) функциональных групп в удерживание сорбата. Так, например, в ряду псевдогомологов, ТАГ: $Л_{3}-Л_{2} \mathrm{O}-Л_{2}-\mathrm{O}_{3}$, по идее об аддитивности в логарифмах факторов удерживания, как параметрах, пропорциональных энергии Гиббса, должно выполняться равенство инкрементов последовательной замены радикалов кислот - линолевой на олеиновую:

$$
\Delta(Л \rightarrow \mathrm{O})=\operatorname{lgk}\left(Л_{2} \mathrm{O}\right)-\operatorname{lgk}\left(Л_{3}\right)=\operatorname{lgk}\left(\text { ЛО }_{2}\right)-\operatorname{lgk}\left(Л_{2} \mathrm{O}\right)=\operatorname{lgk}\left(\mathrm{O}_{3}\right)-\operatorname{lgk}\left(\text { ЛО }_{2}\right) .
$$

Отметим, что упоминание изобарно-изотермического потенциала кажется не совсем уместным для эксперимента, в котором давление на входе и на выходе их колонки существенно различается. Однако, как показывает эксперимент, снижение скорости подачи подвижной фазы приводит к уменьшению разности входного и выходного давления без изменения фактора удерживания, что оправдывает использование термина «энергия Гиббса».

Соотношение (1) не является строгим законом, но экспериментально косвенно подтверждено во множестве работ, в которых вместо инкрементного подхода использовались другие подобные методы [3-7]. Кстати, это равенство позволяет использовать легко доступный ряд псевдогомологов, например, персиковое масло с легко обнаруживаемыми на хроматограмме ТАГ, - Л 3 , Л $2 \mathrm{O}, Л_{2} \mathrm{O}_{2}$ и О 3 , рис. 3 , для определения «мертвого» времени хроматографической системы по уравнению И.Г. Зенкевича [8] вместо полагающегося, но труднодоступного ряда истинных гомологов - ТАГ, образованных только насыщенными жирными кислотами. При этом также легко обнаруживаемые на этой хроматограмме пики ТАГ; Л 2 П, ЛОП, $\mathrm{O}_{2} П$ и $\mathrm{O}_{2} \mathrm{C}$ позволяют рассчитать для данных хроматографических условий ключевые инкременты, табл. 1:

$$
\begin{gathered}
\Delta(Л \rightarrow \mathrm{O})=\operatorname{lgk}\left(\Omega_{2} \mathrm{O}\right)-\operatorname{lgk}\left(\Omega_{3}\right), \\
\Delta(\mathrm{O} \rightarrow \Pi)=\operatorname{lgk}\left(\Omega_{2} \Pi\right)-\operatorname{lgk}\left(\Omega_{2} \mathrm{O}\right), \\
\text { и } \Delta(\Pi \rightarrow \mathrm{C})=\operatorname{lgk}\left(\mathrm{O}_{2} \mathrm{C}\right)-\operatorname{lgk}\left(\mathrm{O}_{2} \Pi\right) .
\end{gathered}
$$

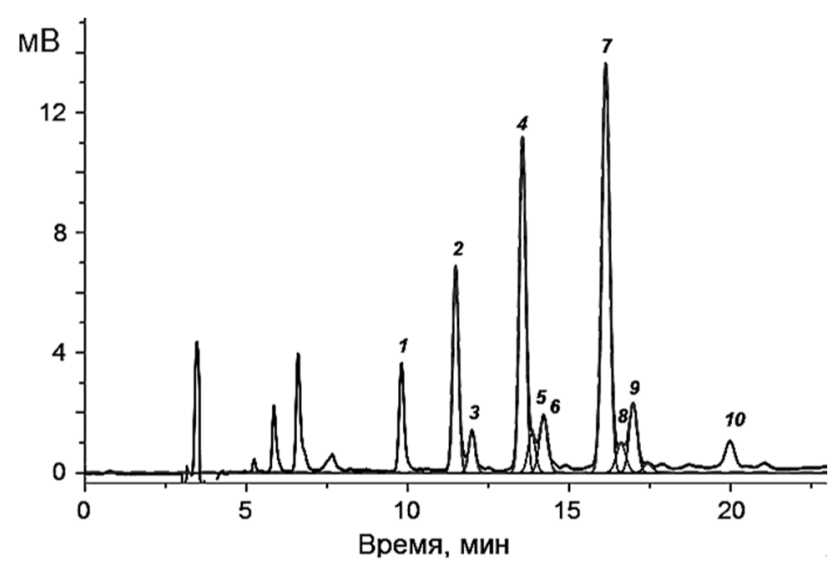

Рис. 3. Хроматограмма персикового масла.

Условия: см. подписи к рис. 2. ТАГ: $1-Л_{3} ; 2-Л_{2} \mathrm{O} ; 3-Л_{2} \Pi ; 4-Л_{2} ; 5-Л_{2} \mathrm{C} ; 6-$ ЛОП; $7-\mathrm{O}_{2}$; $8-$ ЛОС; $9-\mathrm{O}_{2} \Pi ; 10-\mathrm{O}_{2} \mathrm{C}$.

Таблица 1. Параметры удерживания ТАГ персикового масла

\begin{tabular}{|c|c|c|c|c|c|c|}
\hline \multirow{2}{*}{$№$} & \multirow{2}{*}{ ТАГ } & \multirow{2}{*}{$\mathrm{t}_{\mathrm{R}}$, мин } & \multirow{2}{*}{$\operatorname{lgk}(\mathrm{i})$} & \multicolumn{3}{|c|}{ Инкременты } \\
\cline { 5 - 7 } & & 3 & 4 & $\Delta_{2}=\Delta(Л \rightarrow \mathrm{O})$ & $\Delta_{2}=\Delta(\mathrm{O} \rightarrow \Pi)$ & $\Delta_{2}=\Delta(\Pi \rightarrow \mathrm{C})$ \\
\hline 1 & 2 & 3 & 5 & 6 & 7 \\
\hline 1 & $Л_{3}$ & 9.81 & 0.339 & & & \\
\hline 2 & $Л_{2} \mathrm{O}$ & 11.49 & 0.436 & $0.097(1 \rightarrow 2)$ & & \\
\hline 3 & $Л_{2} \Pi$ & 12.00 & 0.462 & & $0.026(2 \rightarrow 3)$ & \\
\hline
\end{tabular}




\begin{tabular}{|c|c|c|c|c|c|c|}
\hline 1 & 2 & 3 & 4 & 5 & 6 & 7 \\
\hline 4 & $\mathrm{JO}_{2}$ & 13.56 & 0.532 & $0.096(2 \rightarrow 4)$ & & \\
\hline 5 & $J_{2} \mathrm{C}$ & 13.90 & 0.546 & & & $0.084(3 \rightarrow 5)$ \\
\hline 6 & ЛОП & 14.22 & 0.558 & $0.096(3 \rightarrow 6)$ & $0.026(4 \rightarrow 6)$ & \\
\hline 7 & $\mathrm{O}_{3}$ & 16.18 & 0.629 & $0.097(4 \rightarrow 7)$ & & \\
\hline 8 & ЛОС & 16.62 & 0.643 & $0.097(5 \rightarrow 8)$ & & $0.085(6 \rightarrow 8)$ \\
\hline 9 & $\mathrm{O}_{2} \Pi$ & 17.00 & 0.655 & $0.097(6 \rightarrow 9)$ & $0.026(7 \rightarrow 9)$ & \\
\hline 10 & $\mathrm{O}_{2} \mathrm{~S}$ & 20.00 & 0.740 & & & $0.085(9 \rightarrow 10)$ \\
\hline & & & Среднее: & 0.097 & 0.026 & 0.085 \\
\hline
\end{tabular}

Относительно количественного анализа ТАГ следует учитывать, что чувствительность детектирования масел зависит от их ненасыщенности, но лаборатории НИУ БелГУ было предложено введение поправок на чувствительность по каждому из ТАГ - по разности между экспериментальным значением коэффициента преломления подвижной фазы и расчетным значением коэффициента преломления соответствующего ТАГ [9].

Если ТАГ масла образованы радикалами пяти кислот, то для полного описания параметров удерживания всех возможных ТАГ достаточно указать:

a) время удерживания реперного соединения, входящего в состав анализируемого масла, удобно использовать в качестве такого реперного вещества трилинолеат, $Л_{3}$, как вещество, входящее в состав ТАГ большого числа растительных масел. Положение Лз на хроматограме исследуемого масла определяют, сравнивая его хроматограмму с хроматограммой персикового масла;

б) три определенных ранее инкремента: $\Delta(Л \rightarrow \mathrm{O}), \Delta(\mathrm{O} \rightarrow \Pi) \Delta(\Pi \rightarrow \mathrm{C})$, поскольку линолевая, олеиновая, пальмитиновая и стеариновая кислоты всходят в состав ТАГ подавляющего большинства растительных масел, и определить значение $\Delta(Л \rightarrow X)$, в котором компонент $\mathrm{X}$ часто является специфическим маркером конкретного масла.

Именно такой подход предлагаем использовать при формировании рабочего листа в программе MS Excel для обработки хроматограммы масла нигеллы дамасской, уникальность которой состоит в том, что в состав ее ТАГ по литературным данным [9] входят радикалы уникальной дигомолиноленовой $\left(\mathrm{C} 20: 2^{11 \mathrm{Z} 15 \mathrm{Z}}\right)$ кислоты (которую обозначим буквой $\mathrm{X})$.

Лист на пятикомпонентный (по числу радикалов различных жирных кислот) состав масел:

а) в столбце (А) нумеруем ТАГ;

б) в столбце (В) (с отступом в 9 строк) перебираем все возможные комбинации радикалов кислот (без дифференциации позиционных изомеров), см. Табл. 1.

в) столбец (C) резервируем для временен удерживания ТАГ с указанием в ячейке С9 «мертвого» времени хроматографической системы:

- для Лз записывается экспериментально определенное по хроматограмме время,

- для остальных ячеек время удерживания рассчитывается по логарифмам факторов удерживания: $\mathrm{C} i=\mathrm{C} 9 *\left(10^{\wedge}(\mathrm{D} i)+1\right)$

г) столбец (D) будет содержать логарифмы факторов удерживания всех ТАГ, построенные по инкрементному подходу, определенные, например, по формулам, приведенным в табл. 2, с использованием инкрементов, занесенных в ячейки:

- $\Delta_{1}=\Delta(Л \rightarrow \mathrm{X})-$ в E1,

- $\Delta_{2}=\Delta(Л \rightarrow \mathrm{O})-$ в E2,

- $\Delta_{3}=\Delta(\mathrm{O} \rightarrow \Pi)-$ в Е3 и

- $\Delta_{4}=\Delta(\Pi \rightarrow \mathrm{C})-$ в E4; 
- после записи формул для расчетов и указания инкрементов в столбце D появятся численные значения логарифмов факторов удерживания, а в столбце C времена удерживания.

- скопировав два столбца В и С, можно вставить их в другой лист, и автоматической сортировкой расположить по возрастанию времен удерживания для удобств в распознавании веществ в смеси по временам удерживания на хроматограмме.

Таблица 2. Параметры для заполнения рабочего листа Excel для масла, ТАГ которого образованы радикалами пяти различных жирных кислот

\begin{tabular}{|c|c|c|c|c|c|c|c|}
\hline № & ТАГ & $\begin{array}{c}\text { Расчет } \\
\lg \boldsymbol{k}(i) \\
\end{array}$ & $\mathrm{n}_{\mathrm{D}}$ расчетный & № & ТАГ & $\begin{array}{c}\text { Расчет } \\
\lg \boldsymbol{k}(i) \\
\end{array}$ & $\begin{array}{l}\mathrm{n}_{\mathrm{D}} \text { рас- } \\
\text { четный }\end{array}$ \\
\hline 1 & $\mathrm{X}_{3}$ & $=\mathrm{B} 26+3 \Delta_{1}$ & 1.488 & 19 & $\Omega_{2} \mathrm{C}$ & $=\mathrm{B} 28+\Delta_{4}$ & 1.480 \\
\hline 2 & $\mathrm{X}_{2} \Omega$ & $=\mathrm{B} 26+2 \Delta_{1}$ & 1.488 & 20 & $\mathrm{JO}_{2}$ & $=\mathrm{B} 27+\Delta_{2}$ & 1.480 \\
\hline 3 & $\mathrm{X}_{2} \mathrm{O}$ & $=\mathrm{B} 12+\Delta_{2}$ & 1.484 & 21 & ЛОП & $=\mathrm{B} 30+\Delta_{3}$ & 1.477 \\
\hline 4 & $\mathrm{X}_{2} \Pi$ & $=\mathrm{B} 13+\Delta_{3}$ & 1.489 & 22 & ЛОС & $=\mathrm{B} 31+\Delta_{4}$ & 1.477 \\
\hline 5 & $\mathrm{X}_{2} \mathrm{C}$ & $=\mathrm{B} 14+\Delta_{4}$ & 1.480 & 23 & ЛП 2 & $=\mathrm{B} 31+\Delta_{3}$ & 1.473 \\
\hline 6 & $\mathrm{X} Л_{2}$ & $=\mathrm{B} 26+\Delta_{1}$ & 1.488 & 24 & ЛПС & $=\mathrm{B} 33+\Delta_{4}$ & 1.473 \\
\hline 7 & ХЛО & $=\mathrm{B} 16+\Delta_{2}$ & 1.484 & 25 & $\pi \mathrm{C}_{2}$ & $=\mathrm{B} 34+\Delta_{4}$ & 1.473 \\
\hline 8 & ХЛП & $=\mathrm{B} 17+\Delta_{3}$ & 1.481 & 26 & $\mathrm{O}_{3}$ & $=\mathrm{B} 30+\Delta_{2}$ & 1.477 \\
\hline 9 & ХЛС & $=\mathrm{B} 18+\Delta_{4}$ & 1.480 & 27 & $\mathrm{O}_{2} \Pi$ & $=\mathrm{B} 36+\Delta_{3}$ & 1.473 \\
\hline 10 & $\mathrm{XO}_{2}$ & $=\mathrm{B} 17+\Delta_{2}$ & 1.484 & 28 & $\mathrm{O}_{2} \mathrm{C}$ & $=\mathrm{B} 37+\Delta_{4}$ & 1.473 \\
\hline 11 & $\mathrm{XO \Pi}$ & $=\mathrm{B} 17+\Delta_{3}$ & 1.481 & 29 & $\mathrm{O \Pi}_{2}$ & $=\mathrm{B} 37+\Delta_{3}$ & 1.469 \\
\hline 12 & $\mathrm{XOC}$ & $=\mathrm{B} 17+\Delta_{4}$ & 1.48 & 30 & ОПС & $=\mathrm{B} 39+\Delta_{4}$ & 1.469 \\
\hline 13 & $\mathrm{X} \Pi_{2}$ & $=\mathrm{B} 21+\Delta_{3}$ & 1.473 & 31 & $\mathrm{OC}_{2}$ & $=\mathrm{B} 40+\Delta_{4}$ & 1.469 \\
\hline 14 & ХПС & $=\mathrm{B} 23+\Delta_{4}$ & 1.473 & 32 & $\Pi_{3}$ & $=\mathrm{B} 39+\Delta_{3}$ & 1.464 \\
\hline 15 & $\mathrm{XC}_{2}$ & $=\mathrm{B} 24+\Delta_{4}$ & 1.473 & 33 & $\Pi_{2} \mathrm{C}$ & $=\mathrm{B} 42+\Delta_{4}$ & 1.465 \\
\hline 16 & $\Omega_{3}$ & & 1.488 & 34 & $\Pi \mathrm{C}_{2}$ & $=\mathrm{B} 43+\Delta_{4}$ & 1.465 \\
\hline 17 & $\mathrm{~J}_{2} \mathrm{O}$ & $=\mathrm{B} 26+\Delta_{2}$ & 1.484 & 35 & $\mathrm{C}_{3}$ & $=\mathrm{B} 44+\Delta_{4}$ & 1.465 \\
\hline 18 & $\pi_{2} \Pi$ & $=\mathrm{B} 27+\Delta_{3}$ & 1.481 & & & & \\
\hline
\end{tabular}

д) в столбец (Е) переносят экспериментально определенные площади пиков с расчетными поправками для неразделенных пиков;

е) столбец (F) содержит расчетные значения коэффициентов преломления ТАГ и экспериментальное значение коэффициента преломления подвижной фазы (в ячейке F9)

ё) в столбце $(\mathrm{G})$ рассчитываем коэффициенты чувствительности рефрактометрического детектора на компоненты $\mathrm{G} i$, приняв за 1 коэффициент чувствительности по Л Л $_{3} \mathrm{G} i=(\mathrm{F} i-\$ \mathrm{~F} \$ 9) /(\$ F \$ 25-\$ F \$ 9) ;$

ж) в столбце $(\mathrm{H})$ рассчитываем исправленные значения площадей пиков: $\mathrm{H} i=\mathrm{E} i / \mathrm{G} i)$;

3) в столбце (I) рассчитываем мольные доли видов ТАГ с учетом суммы всех чисел в столбце $\mathrm{H}:\left(\mathrm{I} i=\mathrm{Hi} / \$ \mathrm{H} \$ 45^{*} 100\right), \%$;

и) в верхних ячейках столбца $G$ записываем символы кислот, образовавших ТАГ исследуемого масла: Х (G1); Л (G2); О (G3); П (G4); С (G5);

к) в ячейках F1-F5 записываем формулу для оценки содержания радикалов каждой из кислот по составу ТАГ и мольной доле такого ТАГ, например, для олеиновой кислоты формула: $=3 * \mathrm{I} 35+2 *(\mathrm{I} 19+\mathrm{I} 29+\mathrm{I} 36+\mathrm{I} 37)+\mathrm{I} 12+\mathrm{I} 16+\mathrm{I} 20+\mathrm{I} 21+\mathrm{I} 26+\mathrm{I} 30+\mathrm{I} 31+$ I38+I39+I40; и в ячейке F6 рассчитываем сумму для всех кислот;

л) в ячейках G1-G5 рассчитываем так называемый жирнокислотный состав масла: $\mathrm{Gi}=\mathrm{F} 1 / \$ \mathrm{~F} \$ 6 * 100, \%$. 
Полученный лист может быть использован как готовый шаблон для дополнительной обработки любых масел, ТАГ которого образованы пятью различными жирными кислотами. Аналогично может быть создан лист для дополнительной обработки любого количества жирных кислот в составе ТАГ масла.

Подбор инкремента $\Delta=\Delta(Л \rightarrow \mathrm{X})$ не вызывает затруднений - на хроматограмме масла нигеллы появляется пик с необычной локализацией на хроматограмме (пик №2 на рис. 4). Его локализация совпадает с известной информацией о присутствии в ТАГ данного масла радикалов $20: 2^{11 Z 14 Z}$ кислоты, для которой инкремент $\Delta_{1}=\Delta(Л \rightarrow \mathrm{X})$ должен быть меньше инкремента $\Delta_{1}=\Delta(\Pi \rightarrow C)$ вследствие добавления двух метиленовых групп во внутреннюю часть радикала примерно на 0.011 логарифмических единиц.

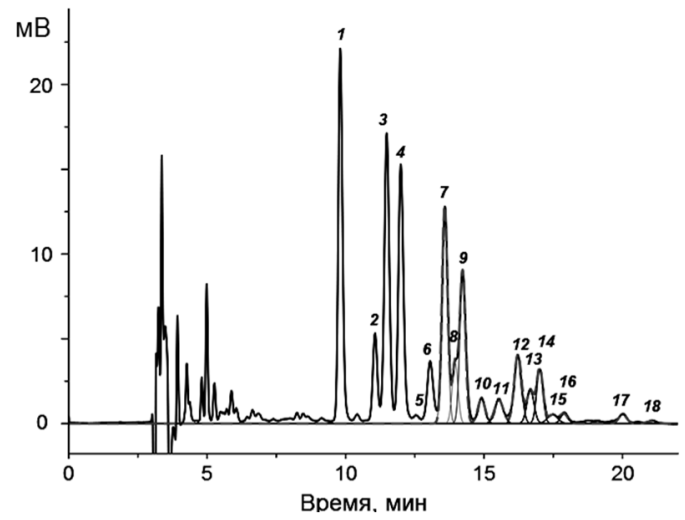

Рис. 4. Хроматограмма масла семян нигеллы дамасской Условия: см. подписи к рис. 2. Отнесение пиков ТАГ см. табл. 3.

Таблица 3. Параметры удерживания ТАГ масла семян нигеллы дамасской

\begin{tabular}{|c|c|c|c|c|c|c|c|c|}
\hline \multirow{2}{*}{ №* } & \multirow{2}{*}{ ТАГ } & \multirow{2}{*}{$\mathrm{t}_{\mathrm{R}}$, мин } & \multirow{2}{*}{$\lg \boldsymbol{k}(i)$} & \multicolumn{4}{|c|}{ Инкременты } & \multirow{2}{*}{$\alpha^{* *}, \%$} \\
\hline & & & & $\Delta(Л \rightarrow \mathrm{O})$ & $\Delta(\mathrm{O} \rightarrow \Pi)$ & $\Delta(Л \rightarrow X)$ & $\Delta(\Pi \rightarrow \mathrm{C})$ & \\
\hline 1 & $\pi_{3}$ & 9.81 & 0.339 & & & & & 14.7 \\
\hline 2 & $\mathrm{X} \pi_{2}$ & 11.06 & 0.413 & & & 0.074 & & 4.0 \\
\hline 3 & $\pi_{2} \mathrm{O}$ & 11.49 & 0.436 & 0.097 & & & & 14.4 \\
\hline 4 & $\pi_{2} \Pi$ & 12.00 & 0.462 & & 0.026 & & & 13.1 \\
\hline 5 & $\mathrm{X}_{2}$ Л & 12.54 & & & & & & 0.8 \\
\hline 6 & ХЛО & 13.05 & 0.510 & 0.097 & & 0.074 & & 3.8 \\
\hline \multirow[t]{2}{*}{7} & $\mathrm{JO}_{2}$ & 13.59 & 0.533 & 0.097 & & & & 9.2 \\
\hline & ХЛП & 13.67 & & & & & & 3.9 \\
\hline 8 & $\pi_{2} \mathrm{C}$ & 13.92 & 0.546 & & & & 0.085 & 3.7 \\
\hline 9 & ЛОП & 14.23 & 0.559 & & 0.026 & & & 9.8 \\
\hline \multirow{2}{*}{10} & $Л_{2}$ & 14.90 & 0.584 & & 0.025 & & & 2.0 \\
\hline & $\mathrm{X}_{2} \mathrm{O}$ & 14.91 & & & & & & \\
\hline \multirow[t]{2}{*}{11} & $\mathrm{XO}_{2}$ & 15.52 & 0.606 & 0.096 & & 0.073 & & 2.2 \\
\hline & $X_{2} \Pi$ & 15.64 & & & & & & \\
\hline \multirow[t]{2}{*}{12} & $\mathrm{O}_{3}$ & 16.21 & 0.630 & 0.097 & & & & 3.8 \\
\hline & ХOП & 16.32 & & & & & & 1.7 \\
\hline 13 & ЛОС & 16.66 & 0.644 & 0.098 & & & 0.086 & 3.0 \\
\hline \multirow[t]{2}{*}{14} & $\mathrm{O}_{2} \Pi$ & 17.01 & 0.655 & & 0.026 & & & 4.3 \\
\hline & $X \Pi_{2}$ & 17.14 & & & & & & \\
\hline 15 & ЛПС & 17.45 & 0.669 & & 0.025 & & & 1.3 \\
\hline 16 & $\mathrm{O \Pi}_{2}$ & 17.89 & 0.682 & & 0.027 & & & 4.3 \\
\hline \multirow[t]{2}{*}{17} & $\mathrm{O}_{2} \mathrm{C}$ & 20.01 & 0.740 & 0.096 & & & 0.085 & 3.1 \\
\hline & ХПС & 20.18 & & & & & & \\
\hline 18 & ОПС & 21.07 & 0.76648 & & 0.026 & & & 1.2 \\
\hline \multicolumn{4}{|c|}{ Среднее значение: } & 0.097 & 0.026 & 0.074 & 0.085 & \\
\hline
\end{tabular}

\footnotetext{
*_номера пиков на рис. 4 ; **- молярная доля ТАГ в масле.
} 
Добавление $\Delta_{1}=\Delta(Л \rightarrow \mathrm{X})=0.074$ позволяет получить параметры удерживания пиков основных ТАГ, близкие к предсказываемым по инкрементному подходу. Расчетные значения долей индивидуальных ТАГ представлены в табл. 3, а расчетный жирнокислотный состав масла составляет: дигомолинолевой кислоты -5.7 , линолевой 49.7 , олеиновой -27.7 , пальмитиновой 12.8 и стеариновой 4.1 моль \%. Полученные в работе результаты трудно сопоставлять с литературными данными, в которых наблюдается очень большой разброс по жирнокислотному составу [10-13]. Однако, в работе [13], в выводах которой отсутствует 20:2 ${ }^{11 Z 14 Z}$ кислота, приведенная хроматограмма позволяет усомниться в правильности выводов авторов.

\title{
Заключение
}

Для определения времени удерживания ТАГ в случае выхода на начальном участке сорбции за пределы линейности в ряде случаев следует использовать время, соответствующее положению вертикали, делящей основание пика пополам. Использование предложенного рабочего листа в MS Excel позволяет быстро и просто определять качественный и количественный состав ТАГ масла по инкрементному подходу и с использованием поправочных коэффициентов для площадей пиков по предложенной в работе процедуре.

\section{Список литературы}

1. Дейнека В.И. // Ж. физ. химии. 2008. Т. 82. 8. Дейнека В.И., Буржинская Т.Г., Дейнека № 1. С. 120-124.

2. Дейнека В.И., Староверов В.М., Фофанов Г.М. и др. // Хим.-фарм. ж. 2002. Т. 36. № 7. C. 44-47.

3. Herslöf B.F., Podlaha O., Töregard B. et al. // J. Am. Oil Chem. Soc. 1979. Vol. 56. pp. 864866.

4 Podlaha O., Töregård B. // J. High Resol. Chromat. 1982. Vol. 5. pp. 553-558.

5 Sempore J.B.G. // J. Chromat. 1986. Vol. 366. pp. 261-282.

6. Lin J., Snyder L.R., Mckeon T.A. // J. Chromat. A. 1998. Vol. 808. pp. 43-49.

7. Takahashi K., Zama K. // J. Am. Oil Chem. Soc. 1985. Vol. 62. pp. 1489-1492.

Л.А. // Аналитика и контроль. 2019. Т. 23. С. 501-508.

9. В.И. Дейнека, Л.А. Дейнека, Н.Г. Габрук. Г.М. и др. // Ж. аналит. химии. 2003. Т.58. С. 1294-1299.

10. Daukšas E., Venskutonis P.R., Sivik B. // J. Food Sci. 2002. Vol. 67. pp. 1021-1024.

11. Telci I., Sahin-Yaglioglu A., Eser F. et al. // J. Am. Oil Chem. Soc. 2014. Vol. 91. pp. 17231729.

12. Parhizkar S., A Latiff L., Abd Rahman S. // Scientific Research and Essays. 2011. Vol. 6. pp. 6817-6820.

13. Khoddami A., Ghazali H.M., Yassoralipour A. et al. // J. Am. Oil Chem. Soc. 2011. Vol. 88. pp. 533-540.

\section{Utilization of MS Excel for processing of vegetable oils chromatograms}

\author{
(C) 2020 Deineka V.I. ${ }^{1}$, Turtygin A.V. ${ }^{2}$, Nguyen Van Anh ${ }^{3}$, Deineka L.A. ${ }^{1}$ \\ ${ }^{1}$ Belgorod National Research University, Belgorod \\ ${ }^{2}$ The National Institute of Accreditation, Moscow \\ ${ }^{3}$ Hanoi Pedagogical University 2, Hanoi, Vietnam
}

A method for the determination of the species composition of triacylglycerines (TAG) and fatty acid composition of vegetable oils was proposed using an incremental approach with simple and accessible computer technologies, specifically, a standard MS Excel worksheet. The qualitative determination is based on the additivity of the contributions of functional groups (fatty acid radicals) into the total retention of TAG and their independence from the structure of the other two radicals in the studied TAG pair. To implement the incremental approach, a method was proposed to specify the TAG retention time beyond the linearity of the sorption 
isotherm. In this case, the best way to determine the retention time is to find the position of the vertical line dividing the peak width into two equal parts. In this work, we used a method for the determination of the "dead" time by retention of series of pseudo-homologues, i.e. TAG groups, with successive replacement of the radicals of one acid for the radicals of another acid. For example, this was implemented with peach oil for the retention times of TAG: $\mathrm{L}_{3}-\mathrm{L}_{2} \mathrm{O}-\mathrm{LO}_{2}-\mathrm{O}_{3}$ according to the previously proposed scheme based on the recurrent relations of I. G. Zenkevich. Following the analysis of the peach oil chromatograms, key increments were calculated for sequential replacement of linoleic-oleic-palmitic-stearic acids. These increments were used for the calculation of the composition of TAG of Nigella damascena seed oil. Quantitative determination of the shares of TAG types required the use of correction factors for experimental values of peak areas due to the dependence of the refractometric detector sensitivity on the difference in refraction coefficients between the mobile phase (experimental data) and the refraction coefficients of individual TAGs calculated in ChemSketch 12.0. The procedure for the arrangement of a worksheet with the indication of the formulas used for calculations is described. The method was used to determine the TAG and fatty acid composition of Nigella damascene L. seed oil. As a result, it was found that the oil contains a unique digomolinoleic acid (C22:211Z14Z).

Keywords: reversed-phase HPLC, resolution of triacylglycerines, qualitative and quantitative analysis, MS Excel worksheet.

\section{References}

1. Deineka V.I., Russ. J. Phys. Chem. A, 2008, Vol. 82, pp. 108-111. https://doi.org/10.1134/ S0036024408010160

2. Deineka V.I., Staroverov V.M., Fofanov G.M. et al., Pharm. Chem, J., 2002, Vol. 36, pp. 392-395. https://doi.org/10.1023/A:1024813 914213

3. Herslöf B.F., Podlaha O. et al. J. Am. Oil Chem. Soc., 1979, Vol. 56, pp. 864866. https://doi.org/10.1007/BF02909537.

4 Podlaha O., Töregard B. J. High Resol. Chromat., 1982, Vol. 5, pp. 553-558. https://doi.org/10.1002/jhrc.1240051007.

5 Sempore J.B.G., J. Chromat., 1986, Vol. 366, pp. 261-282. DOI: 10.1016/s00219673(01)93474-0.

6. Lin J., Snyder L.R., Mckeon T.A., J. Chromat. A, 1998, Vol. 808, pp. 43-49. https://doi.org/10.1016/S0021-9673(98)001344.

7. Takahashi K., Zama K., J. Am. Oil Chem. Soc., 1985, Vol. 62, pp. 1489-1492. https://doi.org/10.1007/BF02541900.

Дейнека Виктор Иванович - профессор кафедры общей химии, д.х.н., Белгородский государственный национальный исследовательский университет, Белгород

Тутыгин Александр Владимирович - младший научный сотрудник Федеральное автономное учреждение «Национальный Институт Аккредитации», Москва

Нгуен Ван Ань - аспирант кафедры общей химии Белгородский государственный национальный исследовательский университет, Белгород

Дейнека Людмила Александровна - доцент кафедры общей химии, к.х.н., Белгородский государственный национальный исследовательский университет, Белгород

8. Deineka V.I., Burzhinskaya T.G., Deineka L.A. Analytics and control, 2019, Vol. 23, pp 501-508. [in Russ.]

9. Deineka V.I., Deineka L.A., Gabruk N.G. et al. J. Anal. Chem., 2003, Vol. 58, pp 1160-1165. https://doi.org/10.1023/B:JANC.0000008956.07 835.51 .

10. Daukšas E., Venskutonis P.R., Sivik B., J. Food Sci. 2002. Vol. 67, pp. 1021-1024. https://doi.org/10.1111/j.1365-

2621.2002.tb09447.x

11. Telci I., Sahin-Yaglioglu A., Eser F. et al. J. Am. Oil Chem. Soc., 2014, Vol. 91, pp. 17231729. DOI: $10.1007 / \mathrm{s} 11746-014-2513-3$

12. Parhizkar S., A Latiff L., Abd Rahman S., Scientific Research and Essays, 2011, Vol. 6, pp. 6817-6820. DOI: 10.5897/SRE11.469. DOI: 10.5897/SRE11.469

13. Khoddami A., Ghazali H.M., Yassoralipour A. et al., J. Am. Oil Chem. Soc., 2011, Vol. 88, pp. 533-540. https://doi.org/10.1007/s11746$010-1687-6$

Deineka Viktor I. - Professor of the Department of General Chemistry Belgorod state national research University, Belgorod, e-mail deineka@,bsu.edu.ru

Turtygin Alexander V. - Junior researcher Federal Autonomous institution "National Institute of Accreditation", Moscow, e-mail issanea@mail.ru

Nguyen Van Agn - post-graduate of the Department of General Chemistry Belgorod state national research University, Belgorod, e-mail Vananhkhoanosh2gmail.com

Deineka Lyudmila, Associate Professor of the Department of General Chemistry Belgorod state national research University, Belgorod 